\title{
Biomechanical effects of the addition of a precision constraint on a collective load carriage task
}

\author{
$1 \quad$ Nour Sghaier ${ }^{1}$, Guillaume Fumery ${ }^{1}$, Vincent Fourcassié ${ }^{1}$, Nicolas A. Turpin², Pierre \\ 2 \\ Moretto*1 $^{* 1}$ \\ $3 \quad{ }^{1}$ Centre de Recherches Sur La Cognition Animale, Centre de Biologie Intégrative, Université \\ 4 de Toulouse, CRCA, UMR CNRS-UPS 5169, 118 Route de Narbonne, 31062, Toulouse, \\ 5 France
}

$6 \quad{ }^{2}$ IRISSE Lab (EA 4075), UFR SHE, Sport Sciences Department (STAPS), Université de La

7 Réunion, 117, rue du général Ailleret, 97430, le Tampon, France

8 * Correspondence:

9 Pierre Moretto

10 pierre.moretto@univ-tlse3.fr

11 Words count:

12 Abstract: $238(\max =250)$

13 Main Text: $4455(\max =7000)$ 


\section{Abstract}

15 Team lifting is a complex and collective motor task that possesses both motor and cognitive components. The purpose of this study was to investigate to what extent the biomechanics of a collective load carriage is affected when a dyad of individuals is performing a carrying task with an additional accuracy constraint. Ten dyads performed a first condition in which they collectively transported a load (CC), and a second one in which they transported the same load while maintaining a ball in a target position on its top (PC).

The recovery-rate, amplitude, and period of the center-of-mass (COM) trajectory were computed for the whole system $($ dyad + table $=$ PACS $)$. We analyzed the forces and moments exerted at each joint of the upper limbs of the subjects. We observed a decrease in the overall performance of the dyads when the Precision task was added, i.e., i) the velocity and amplitude of CoMpaCs decreased by $1,7 \%$ and $5,8 \%$, respectively, ii) inter-subject variability of the Moment-Cost-Function decreased by $95 \%$ and recovery rate decreased by $19,2 \%$ during PC. A kinetic synergy analysis showed that the subjects reorganized their coordination in the PC.

Our results demonstrate that adding a precision task affects the economy of collective load carriage. Notwithstanding, the joint moments at the upper-limbs are better balanced and covary more across the paired subjects during the precision task. Our study results may find applications in domains such as Ergonomics, Robotics-developments, and Rehabilitation.

Keywords: Ergonomic, Dual Task, Load Carriage, Collective Behavior, Team Lifting, Precision Task. 


\section{Introduction}

Individual manual materials handling is commonly performed in many human activities. Its definition regroups essentially different tasks of load handling (i.e. the transport, support, lift, push, pull...) and has unfavorable ergonomic conditions (Directive 90/269/CEE). Different lifting techniques (Faber et al., 2007) and mechanical aids (Godwin et al., 2009) have been proposed to avoid the associated leisure. However, the most common alternative used is team lifting. It is, in appearance, a simple solution to carry a heavy load (i.e. too heavy to be carried safely by a single individual), a bulky object without mechanical aids. This strategy is then supposed to reduce the load on individuals performing the task (Sharp et al., 1997). It is also used in some sports such as Crossfit discipline when lifting "worm", a heavy, long, and soft cylindrical bag (Claudino et al., 2018).

Furthermore, team lifting necessitates both motor and cognitive skills in order to control the movement coordination. For this reason, many authors consider it as a dual-task, e.g. workdays in ironwork (Faber et al., 2012). The tasks are called dual as they often undergo some interference, linked to a limited ability to share attention between the two task goals. Dual-task interference are commonly studied in psychology to highlight the cognitive limits of the human brain (Pashler, 1994). One well-known example of these cognitive limits is based on locomotion which can be used in interference paradigm (Yogev-Seligmann et al., 2008; YogevSeligmann et al., 2010). For example, walking along an L-shaped path while performing an arithmetic task deteriorates the mobility function. Any additional cognitive-task is considered as a limiting factor for the motor task since it induces a modification of gait pattern e.g., reduces gait speed, induces movement fluctuation and oscillation. Beach et al., (2006), showed that during a repetitive lifting task adding a precision placement challenges leads to an increase of lumbar spine load and an increase of the upper limb movement time.

Recent studies focused on lifting-Precision dual-task and showed that locomotor pattern was not affected when the subjects performed a dual-ask such as carrying a load (20\% of mean body mass) on the shoulders (Castillo et al., 2014), or carrying a load (21\% and $36 \%$ of mean body mass) on the back(Ackerman and Seipel, 2014; Bastien et al., 2016). However, other studies reported a walking pattern affected by the dual-task when the load is balanced on the top of the head (Heglund et al., 1995) or when it is carried collectively (Fumery et al., 2018). In certain jobs, a collective load transfer is simultaneously associated to a precision task (e.g. talking with the patient in nurses, administrating medication in stretchers bearer, Industrial manufacturing 
in workman). This occurs especially in paramedics or during search and rescue activities (Gamble et al., 1991; Restorff, 2000; Barnekow-Bergkvist et al., 2004; Leyk et al., 2007).

Despite this practical relevance, only few studies deal with biomechanical aspects of collective transport of a load. However, it is essential to conceive aids, exoskeletons and collaborative robots dedicated to assisting humans in such a task. Fumery et al., (2018) studied the energetic exchanges during a collective load carriage to investigate whether two individuals transporting an object behave economically. The authors showed that the external energetic exchanges occurring during this type of transport was as efficient as those occurring in single gait when the load is below $10 \%$ of the total body mass of the dyad. In our study, we reproduce the protocol of Fumery et al. to investigate the locomotor pattern of ten paired individuals carrying a box collectively and to compare these walking patterns when this task is performed simultaneously with a precision task. This precision task consists in maintaining a ball in the center of a circular target drawn on the top of the box. Thanks to handle sensors and kinematic data, we also record the forces and moments applied on the box then compute the constraints at the arms and back joints using the inverse dynamics bottom-up procedure. The purpose of this study is to investigate to what extent the performance of a collective load transport is impacted when a cognitive task is performed simultaneously, and how the control of the cognitive task is shared across the subjects. As it has been observed in single subjects walking and performing a cognitive task, we hypothesize that gait performance of the individuals walking while transporting collectively a load is disturbed when they perform a precision task requiring precision. Fumery et al. (2018) demonstrating that the pattern of a dyad carrying a light load was not affected by the collective task, our hypothesis is that, using the same load ratio, the precision task is the single factor affecting the walking-carrying pattern of the paired subjects.

\section{$3 \quad$ Materials and Methods}

\subsection{Population}

Ten pairs of healthy male individuals (mean \pm s.d.: volunteer 1 - at the left side of the load: height $=1.77 \pm 0.07 \mathrm{~m}$, mass $=74.78 \pm 9.00 \mathrm{~kg}$; volunteer 2 - at the right side of the load: height $=$ $1.77 \pm 0.05 \mathrm{~m}$, mass $=74.54 \pm 12.38 \mathrm{~kg}$ ) participated in the experiments. The individuals had no orthopedic disabilities, no dysfunctions of the locomotor system, no neurological or vestibular diseases, no visual deficits and no proprioceptive disorders or dementia.

This study was carried out in accordance with the requirement of a non-interventional study given by the CNRS bioethical office. The study was approved by the Research Ethics 
Committee of the University of Toulouse, France (number IRB00011835-2019-11-26-172, Université Fédérale de Toulouse IRB \#1). All subjects gave verbal and written informed consent in accordance with the Declaration of Helsinki.

\subsection{Experimental Protocol}

Two conditions were performed by the subjects. In the first condition (Control Condition: CC) they walked side by side at spontaneous speed while carrying a box (mass $=13.41 \mathrm{~kg}$, size: 0.40x0.40x0.28 m) equipped with two lateral handle sensors (Sensix, France). The mass of the box plus the sensors was $14.250 \mathrm{~kg}$ thus almost $10 \%$ of the body-mass of the two volunteers. In order to get accustomed to the task the subjects performed three successive trials. Only the last and third trial was retained for the analysis.

In the second condition (Precision Condition: PC), the individuals were instructed to transport the box, while performing an accuracy task consisting in keeping a ball (diameter: $19 \mathrm{~mm}$, mass: $2 \mathrm{~g}$ ) in the center of a circular target drawn on the top of the box (Fig 1). Subjects were not allowed to orally communicate during the experiments.

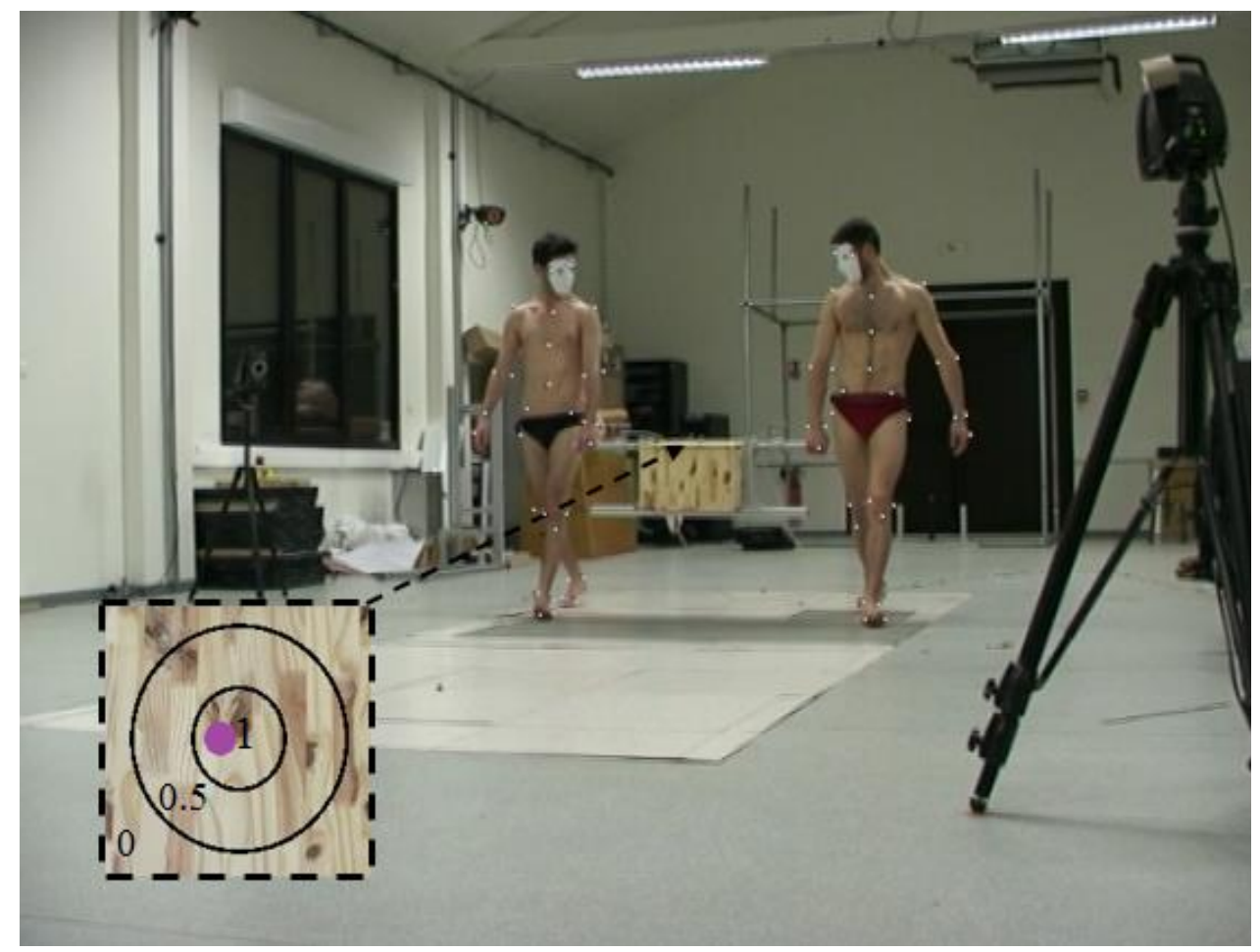



with $G_{P A C S}$ the 3D position of the CoMpaCs in the frame R (the global coordinate system), $m_{P A C S}$ the mass of the PACS, $n$ the number of PACS segments (i.e. 16 segments per volunteer

obtained a 1-point Scorep. If it was maintained between the small and large circle, then they obtained 0,5-point Scorep. Else, they obtained 0-point Scorep.

\subsection{Kinematic and Kinetic Data Acquisition}

Motion capture data were collected using thirteen infrareds (11 MX3 and 2 TS40) transmitterreceiver video cameras $\left(\right.$ Vicon $^{\odot}$, Oxford metric's, Oxford, United Kingdom) sampled at 200 Hz. Forty-two retro-reflective markers were placed on bony landmarks and on the navel of each subject ( according to Wu et al., 2002, 2005) and fourteen on the box. The ball used during the PC tests was reflective as well and was tracked by the Vicon ${ }^{\odot}$ system.

In order to record the gait pattern at constant speed (i.e. to exclude the acceleration and deceleration phases at the beginning and end of each trial) the volume calibrated by the $\operatorname{Vicon}^{\odot}$ system $\left(30 \mathrm{~m}^{3}\right)$ was located in the middle of the $20 \mathrm{~m}$-long walkway crossed by the subjects. The reflective marks were tracked to define the kinematics of the Poly-Articulated Collective System (PACS) formed by the two individuals and the load they carry (Zatsiorsky, 1983; Moretto et al., 2016). The data were recorded on one gait cycle defined by the first heel strike of the first subject and the third heel strike of the second subject of the PACS to ensure a cycle of each subject. The 3D reconstruction was performed using Vicon Nexus $1.8 .5^{\odot}$ software.

The two lateral handles used to transport the box were equipped with Sensix ${ }^{\circledR}$ force sensors sampled at $2000 \mathrm{~Hz}$. A $4^{\text {th }}$ order Butterworth filter and a $5 \mathrm{~Hz}$ and $10 \mathrm{~Hz}$ cut frequency have been applied to analyze the positions of the markers and the forces exerted on the box handles, respectively.

\subsection{Computed parameters}

\subsubsection{Trajectory of the CoMpacs}

The De Leva Anthropometric tables (de Leva, 1996) was used to estimate the mass $\mathrm{m}_{\mathrm{i}}$ and the $\mathrm{CoM}$ of each segment $\mathrm{i}\left(\mathrm{CoM}_{\mathrm{i}}\right)$ of the PACS and to compute its global CoM (CoMpacs) as follow:

$$
\boldsymbol{G}_{\boldsymbol{P A C S}}=\frac{1}{m_{P A C S}} \sum_{i=1}^{n=33} m_{i} \boldsymbol{G}_{i}
$$
plus one segment for the box) and $\boldsymbol{G}_{\boldsymbol{i}}$ the $3 \mathrm{D}$ position of the $\mathrm{CoM}_{\mathrm{i}}$ in the frame R. The CoM of 
147 the box was determined at the intersection point of the vertical lines obtained by hanging it with

148 a thread fixed at different positions. The material used for the box construction, i.e. wood and

149 aluminium, was considered as not deformable.

150 According to Holt et al., (2003), the amplitude $\left(A=Z_{\max }-Z_{\min }\right.$, with $Z$ the height of the

151 CoMPACS, in meters, ) and the period (peak to peak, in percent of the gait cycle) of the CoMPACS

152 were also assessed.

153 The forward kinetic $\left(W_{\mathrm{kf}}\right)$, as well as the vertical $\left(W_{\mathrm{v}}\right)$ and external work $\left(W_{\mathrm{ext}}\right)$ of the CoMPACS

154 were computed according to the method of Bastien et al. (2016). Then based on the external

155 work, the percentage of energy recovered of the CoMpACs in the sagittal plane was computed

156 (called recovery rate $R R$ in Fumery et al., 2018a, 2018b). This parameter assess the amount of

157 energy transferred between the potential and the kinetic energy (Eqn 2).

$$
R R=100 \frac{W \mathrm{kf}+W \mathrm{v}-W \mathrm{ext}}{W \mathrm{kf}+W \mathrm{v}}
$$

159

160

161

162

163

164

165

166

167

168

169

170

171

172

173

174

175

176

The closer the value of $R R$ to $100 \%$, the more consistent the locomotor pattern is with the inverted pendulum system (IPS) model of locomotion (Cavagna et al., 1963; Willems et al., 1995; Gomeñuka et al., 2014; Bastien et al., 2016). In this study, the trajectory of CoMPACs and $\mathrm{CoM}$ of an inverted pendulum have been investigated.

\subsubsection{Forces and moments at the joints of the upper limbs}

Sensix force sensors recorded the forces and moments applied by each individual on the two box handles. Before the computation, the data of the sensors located by specific markers were transfer to the Galilean frame of the laboratory using rotation matrix. A cross correlation method has been applied in order to analyze the coordination between the forces produced by both subjects. To investigate whether the movement of the box results from an action-reaction strategy, we computed the time lag required for the position of the left side and right side of the box to be the same on the medio-lateral, antero-posterior and vertical axis in CC and PC. The coordination was assessed through the forces exerted on three directions (medio-lateral, anteroposterior and vertical axis). This results will reflect the level of coordination of two subjects during a collective transport

In order to quantify muscular constraints produced at the upper limb, the Inverse Dynamic Method was used to estimate forces and moments at each joint of the upper limb. The Moment Cost Function was then computed $\left(\mathrm{kg} \cdot \mathrm{m}^{2} \cdot \mathrm{s}^{-2}\right.$, Costes et al., 2018) as follow : 


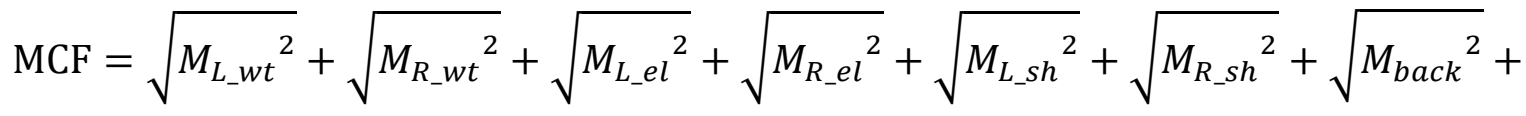

$$
\sqrt{M_{\text {neck }}^{2}}
$$

179 Where $M_{L_{-} w t}, M_{R_{-} w t}, M_{L_{-} e l}, M_{R_{-} e l}, M_{L_{-} s h}, M_{R_{-} s h}, M_{b a c k}$ and $M_{\text {neck }}$ are the mean values over a

180 PACS gait cycle of the three-dimensional left and right wrist, left and right elbow, left and right

181 shoulder, top of the back and neck moments, respectively. $\sqrt{\mathrm{M}^{2}}$ represents the Euclidian norm of $\mathrm{M}$ (i.e. $\sqrt{\mathrm{M}^{2}}=\sqrt{\sum_{i=1}^{3}\left(M_{i}\right)^{2}}$, with $\mathrm{M}_{\mathrm{i}}$ the $\mathrm{i}^{- \text {th }}$ component of the vector $\mathrm{M}$ ).

183 Then, the MCF values of each individual was summed to obtain the total moment cost function

184 (Total $M C F$ ). This Total $M C F$ allows to quantify the global effort produced at the upper-limbs 185 of the PACS during one gait cycle. Finally, the $M C F$ difference ( $\triangle M C F$ ) was computed as the 186 difference between the two individuals to investigate whether the subjects produced the same 187 effort in the upper limbs during the load transport.

\subsubsection{Kinetic synergy analysis}

189 We extracted the synergies by using a principal component analysis (PCA) applied to the wrist, 190 elbow, shoulder, back, and neck joint moment on the right and left sides of the body. The PCA 191 was used to reduce data dimensionality. It consisted in the eigen-decomposition of the co192 variance matrix of the joint moment data (Matlab eig function). The joint moments data from one trial per condition were arranged in time $\times$ joint moment matrices. In this analysis we only used the $y$-component which is very close to the norm of the $3 \mathrm{D}$ joint moments, except that the y-component (medio-lateral) could be positive and negative. The joint moments were normalized by their amplitude and centered (mean removed) before application of the PCA. We called the eigenvectors extracted from the PCA, dynamic synergy vectors. We computed the VAF (Variance Accounted For) which corresponded to the cumulative sum of the eigenvalues, ordered from the greatest to the lowest value, normalized by the total variance computed as the sum of all eigenvalues. The synergy vectors retained were then rotated using a Varimax rotation method to improve interpretability.

We first extracted the synergy vectors for each experimental condition and each participant separately. In this analysis the initial data matrices were constituted of all available time frames in line, concatenated from one trial per condition, and of eight columns corresponding to each joint moment, namely the right wrist, left wrist, right elbow, left elbow, right shoulder, left 
shoulder, back, and neck. Based on a previous study we extracted 3 synergies in this analysis.

207 We then performed a second analysis to identify possible co-variations between the joint moments of the two participants in each pair. The columns of the initial matrices were thus constituted of the joint moments of the two loaded arms, i.e., the right wrist, elbow, and shoulder joint moments of participant \#1, plus the left wrist, elbow and shoulder joint moments of

211 participant \#2. Based on a previous study we extracted 2 synergies in this analysis. We used

212 Pearson's $r$ to order the different synergies similarly between the different subjects and 213 conditions.

\subsubsection{Accuracy score}

215 A performance score ( score $_{\mathrm{p}}$ ) was assigned to each image of the videos captured by the Vicon ${ }^{\odot}$ system (200 images/s). The score depended on the location of the ball in the target: 1 when the ball was inside the small circle, 0.5 when it was in-between the small and large circle and 0 when it was outside the large circle. The accuracy over the whole gait cycle was measured by

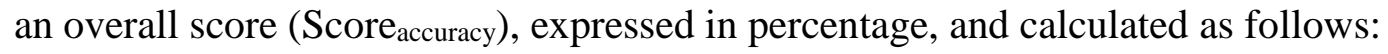

$$
\text { Score }_{\text {accuracy }}=\frac{\sum \text { Score }_{\mathrm{p}} \times \mathbf{1 0 0}}{\mathbf{t}_{\text {gait cycle }}}
$$

where $t_{\text {gait cycle }}$ represents the number of $\mathrm{Vicon}^{\odot}$ images recorded along one gait cycle.

\subsubsection{Orientation of the upper part of the body}

The head, shoulders and pelvis rotation angles were computed around the vertical axis of each individual in the two conditions. The angle was positive when the subjects turned towards the box they carried, otherwise it was negative. The distance between the forehead and the sternum (distance FOR-STE) was also computed in order to investigate the flexion of the cervical spine.

\subsection{Data analysis}

228 The data were analyzed with Matlab R2016b@ and StatView 5.0@ software. A paired t-test was used to compare the RRs, the amplitudes, the periods, the velocities of the vertical displacement

230 of the CoMPACS, the head, shoulders and pelvis rotation angle and the length FOR-STE 231 between the CC and PC condition. The significance threshold was set to 0.05 . We computed 232 with a cross-correlation method the time lag required for the position of the left side and right 233 side of the box to be the same on the medio-lateral, antero-posterior and vertical axis in CC and PC. 
235 We used the average subspace angles to compare the subspaces spanned by the synergy vectors

236 (Knyazev and Argentati, 2002). In order to decide whether the subspaces were more similar 237 than expected by chance, the confidence interval (CI) of random comparisons was computed.

238 For this analysis, we generated pairs of random subspaces constituted each of either 3 unit 239 vectors of dimension 8 (individual PCA analysis) or 2 unit vectors of dimension 6 (conjoint 240 PCA analysis) and computed the mean subspace angle between them. The unit vectors were 241 built using normally distributed pseudo-random numbers (Matlab randn function). We 242 performed 10000 simulations in order to determine the 95\%-CI of the mean subspace angle 243 between the pairs of random subspaces. The confidence interval was $39.5^{\circ}-70.0^{\circ}\left(55.0 \pm 7.6^{\circ}\right)$ 244 for the individual PCA analysis and $36.3^{\circ}-79.1^{\circ}\left(57.7 \pm 10.7^{\circ}\right)$ for the conjoint analysis.

245 We used Student tests for single mean to compare the subspaces angles to the lower bound CI 246 with the assumption that similarity was higher than expected by chance when the angles were 247 lower than the lower bound CI.

248 VAFs were compared with an ANOVA with one repeated measure (control vs. precision 249 conditions) and one factor (participant \#1 vs. participant \#2) when synergies were extracted 250 separately for each subject. For the conjoint analysis, a paired Student t-test was used. Subspace 251 angles were compared with t-tests for dependent samples (paired t-test) when comparing the 252 control and precision conditions and t-test for independent samples when comparing the two 253 participants. Adjustments for multiple comparisons were performed by Bonferroni's method. 254 Initial level of significance was set to $\mathrm{p}<0.05$.

2554 Results

$256 \quad 4.1$ Dynamics analysis of the dual-task

$257 \quad$ 4.1.1 CoM trajectory

258 The CoMpaCs velocity significantly decreased from $1.40 \pm 0.14 \mathrm{~m} . \mathrm{s}^{-1}$ in CC to $1.23 \pm 0.17 \mathrm{~m} . \mathrm{s}^{-1}$ 259 in PC ( $t=3.385, \mathrm{p}=0.008)$. The CoMPACs amplitude (Fig. 2A, $t=3.704, p=0.005)$, significantly 260 decreased from $2.87 \pm 0.742 \mathrm{~cm}$ in $\mathrm{CC}$ to $2.29 \pm 0.739 \mathrm{~cm}$ in PC (Table S3). However, the 261 period of the CoM $262 t=0.842, \mathrm{p}=0.422)$. 
A

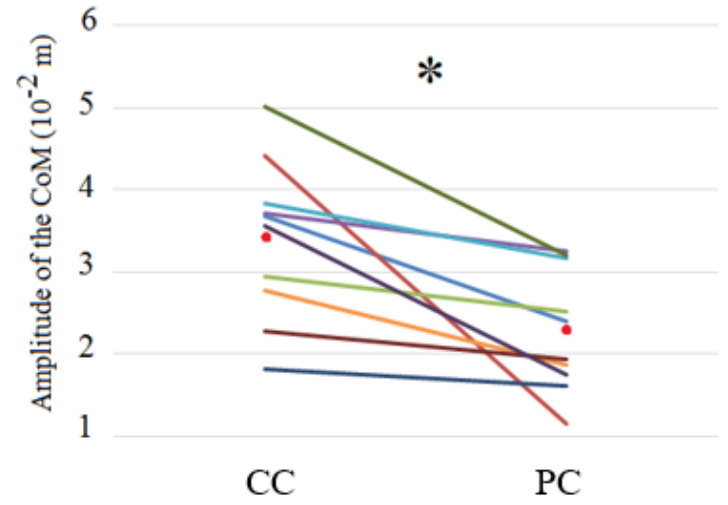

B

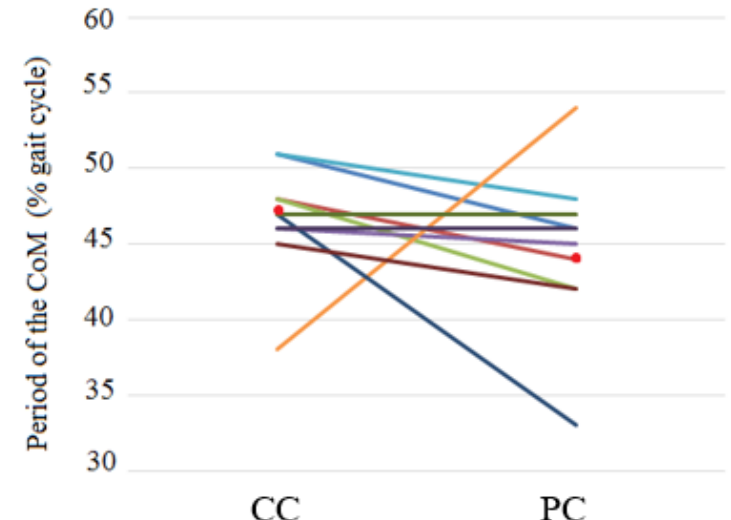

Figure 2. Amplitude (A) and period (B) of the vertical displacement of the CoMPACS in the Control Condition (CC) and the Precision Condition (PC). The mean value of each dyad $(\mathrm{N}=10)$ was computed for the $\mathrm{CC}$ and PC and linked. The red points represent the mean for each condition. The same color is assigned to each dyad in all figures. ${ }^{*}=$ significant difference $(\mathrm{p}<0.05$ paired $\mathrm{t}$-test).

The percentage of energy recovered at the CoMPACS significantly decreased between CC and PC $(t=5.18, p<0.001)$ (Fig 3). This showed an alteration of the efficiency of the locomotor pattern of the dyad when the energy transfer between the potential and the kinetic energy was by $19,2 \%$ lower in PC compared to CC.

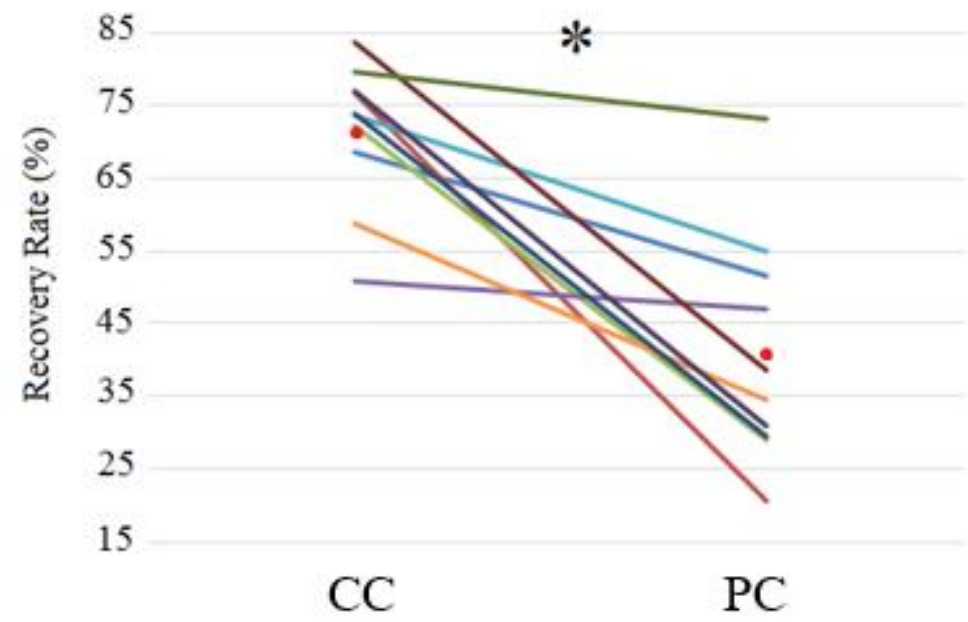
each dyad in all figures. $*=$ significant difference $(\mathrm{p}<0.05$ paired $\mathrm{t}$-test $)$. 


\subsubsection{Forces applied to the handles}

279 Significant differences were found between the correlation coefficients of the three components 280 (x, y, z) of the forces applied to the handles by the subjects of the dyad for each condition. In 281 the CC condition, $\mathrm{R}_{\mathrm{Fx}}$ was lower than $\mathrm{R}_{\mathrm{Fy}}(\mathrm{t}=-3.45, \mathrm{p}<0,001)$ and $\mathrm{R}_{\mathrm{Fz}}(\mathrm{t}=-4.53, \mathrm{p}<0,01)$ and $282 \mathrm{R}_{\mathrm{Fy}}$ was lower than $\mathrm{R}_{\mathrm{Fz}}(\mathrm{t}=-2.48, \mathrm{p}=0,04)$. In the PC condition, $\mathrm{R}_{\mathrm{Fx}}$ was lower than $\mathrm{R}_{\mathrm{Fy}}(\mathrm{t}=-$ 283 6.06, $\mathrm{p}<0,01)$ and $\mathrm{R}_{\mathrm{Fz}}(\mathrm{t}=-4.50, \mathrm{p}<0,01)$. However, no significant differences (Fig. 4A) were 284 found between the $\mathrm{CC}$ and the PC conditions $(\mathrm{t}=-0.43, \mathrm{p}=0.675 ; \mathrm{t}=-1.43, \mathrm{p}=0.188 ; \mathrm{t}=$ $2851.02, \mathrm{p}=0.335$ for the medio-lateral, antero-posterior and vertical axis, respectively) (Table 286 S8).

287 In the $\mathrm{CC}$, the time lags were lower than $150 \mathrm{~ms}$ in the medio-lateral and antero-posterior axis and only one lag was higher than $150 \mathrm{~ms}$ for one dyad for the vertical axis (Table 1, lagZ $20 \pm$ $50 \mathrm{~ms}$ ). Concerning the PC, the time lags were also lower than $150 \mathrm{~ms}$ in the medio-lateral and antero-posterior axis and five dyads had a time lag higher than $150 \mathrm{~ms}$ for the vertical axis
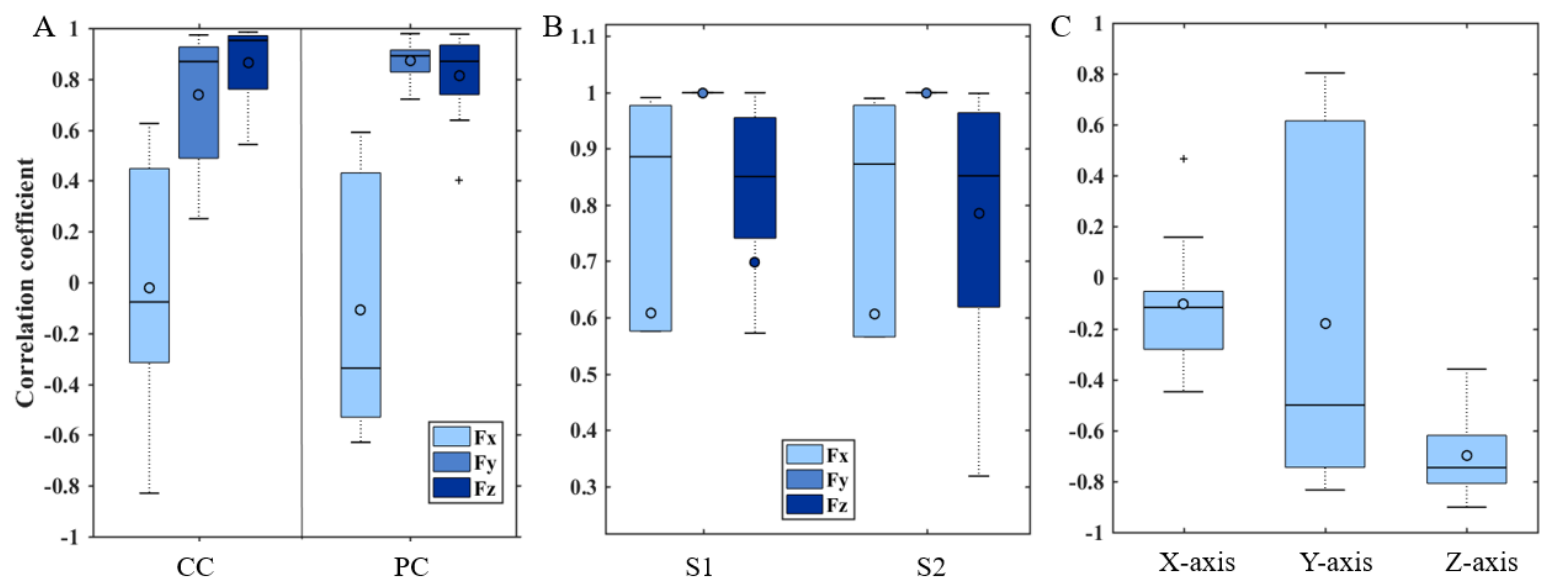

292

Figure 4. Boxes plot showing the distribution of the correlation coefficient (Coef). A : Coef of the forces produced by the individuals in each dyad on the box handles, on the medio-lateral (Fx), anteroposterior (Fy) and vertical axis (Fz) in the CC and PC conditions. B : Coef of the ball displacement and the handles displacement, on Fx, Fy, and Fz, in the CC and PC conditions. C : Coef of the ball trajectory and the sum of forces exerted by the subjects on the handles, on Fx, Fy, and Fz, in PC. N = 10 for each condition. $* 0.05>\mathrm{p}>0.01 ; * * \mathrm{p}<0.01$ (paired Student $\mathrm{t}$ test). The upper horizontal line of the box represents the third quartile (75th percentile), the lower line of the box represent the first quartile (25th percentile), the middle value of the dataset is the median value (50th percentile) and the upper and lower horizontal lines outside the box represent respectively 90 th percentile and 10th percentile. Cross-and circle represent respectively outlier and mean. 


\subsubsection{Kinetic synergy analysis}

304 Consistent with a previous study we extracted 3 dynamic synergies for all subjects, which 305 accounted for $96.3 \pm 2.0 \%$ of total variance on average (range [90.0-99.0]\%). We found no 306 effect of the side (being on the left or right side of the load) nor of the precision constraint on 307 the VAF values (i.e., $|\Delta \mathrm{VAF}|=1.9 \pm 0.4 \%, p$-value $=0.24, \eta^{2}=0.08$ and $|\Delta \mathrm{VAF}|=0.9 \pm 0.5 \%, p$ 308 value $=0.62, \eta^{2}=0.01$, respectively).

309 The dynamic synergies for each participant are depicted in Fig 5. The comparisons between 310 participant \#1 and participant \#2 gave subspaces angles not different than expected by chance 311 in the precision condition (i.e., $45.0 \pm 10.0^{\circ}$ compared to $39.5^{\circ}$, Student $t_{9}=-1.34 ; p$-value $=0.21$; 312 Fig. 6A). For the other comparisons in Fig 6, subspace angles were lower than expected by 313 chance (Fig 6, $\mid$ Student t $\geq 7.6 ; p$-value $<0.001$ ). The subspace angles were lower in the control 314 condition than in the precision condition when comparing participant \#1 and participant \#2 (Fig. 315 6A, Cohen's d=1.9; Student t $t_{9}=-4.95$; $p$-value $<0.001$ ); showing an effect of the conditions on 316 inter-subject similarities. The comparison between the control and precision conditions gave 317 subspace angles of $27.2 \pm 5.7^{\circ}$ on average with no differences between subjects (Fig. 6B, 318 Cohen's $d=0.61$; Student $t_{9}=-1.93 ; p$-value=0.09). The inter-condition subspace angles were 319 lower than inter-subject subspace angles (i.e., $27.2 \pm 5.7^{\circ}$ vs. $37.1 \pm 7.7^{\circ}$, Cohen's $\mathrm{d}=1.5$; Student $\mathrm{t}_{18}=3.30 ; p$-value $\left.=0.004\right)$.

321 For the conjoint PCA analysis, two dynamic synergies were extracted for all pairs of subjects, which accounted for $95.1 \pm 3.5 \%$ of total variance on average (range [84.4-98.8]\%). The dynamic synergies for the conjoint analysis are depicted in Fig 7. VAFs were similar in the control and precision conditions $(96.6 \pm 2.5 \%$ vs. $93.5 \pm 3.8 \%$, respectively, Cohen's $d=0.7$; Student $t_{9}=2.18 ; p$-value $=0.06$ ). The subspace angles were not different than expected by chance (i.e., $46.4 \pm 21.3^{\circ}$ compared to $36.3^{\circ}$, Student $t_{9}=1.50 ; p$-value $=0.17$ ) when comparing the control and precision conditions (Fig. 7B). 


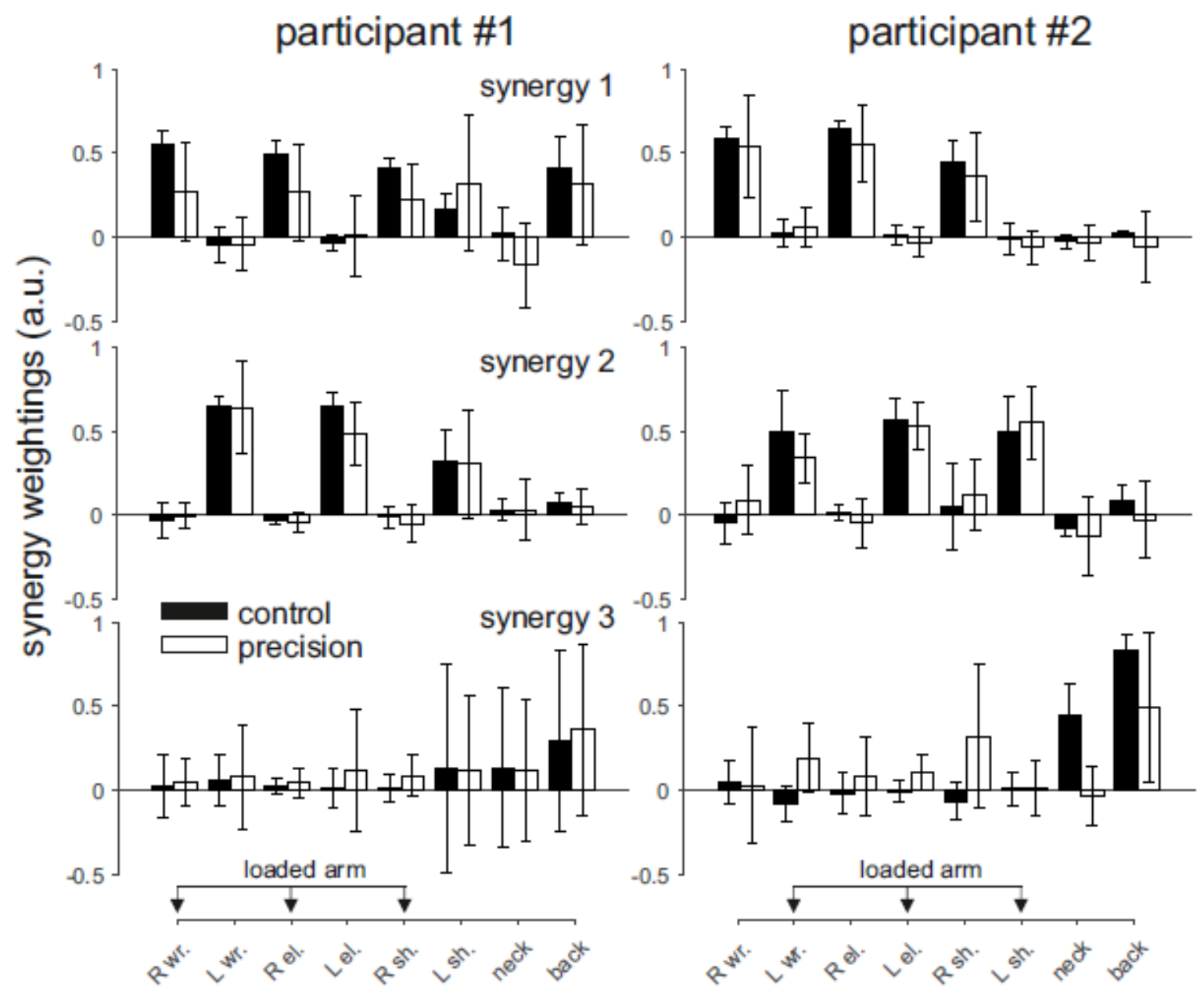

330 Figure 5. Dynamic synergy vectors. Three synergies accounted for more than $90.0 \%$ of total variance in all subjects and conditions. Participants labeled \#1 were on the right side of the load and participants \#2 on the left side. Wrist, elbow and shoulder were abbreviated to wr. el. and sh. respectively. $\mathrm{R}$ and $\mathrm{L}$ refer to right and left side, respectively. 

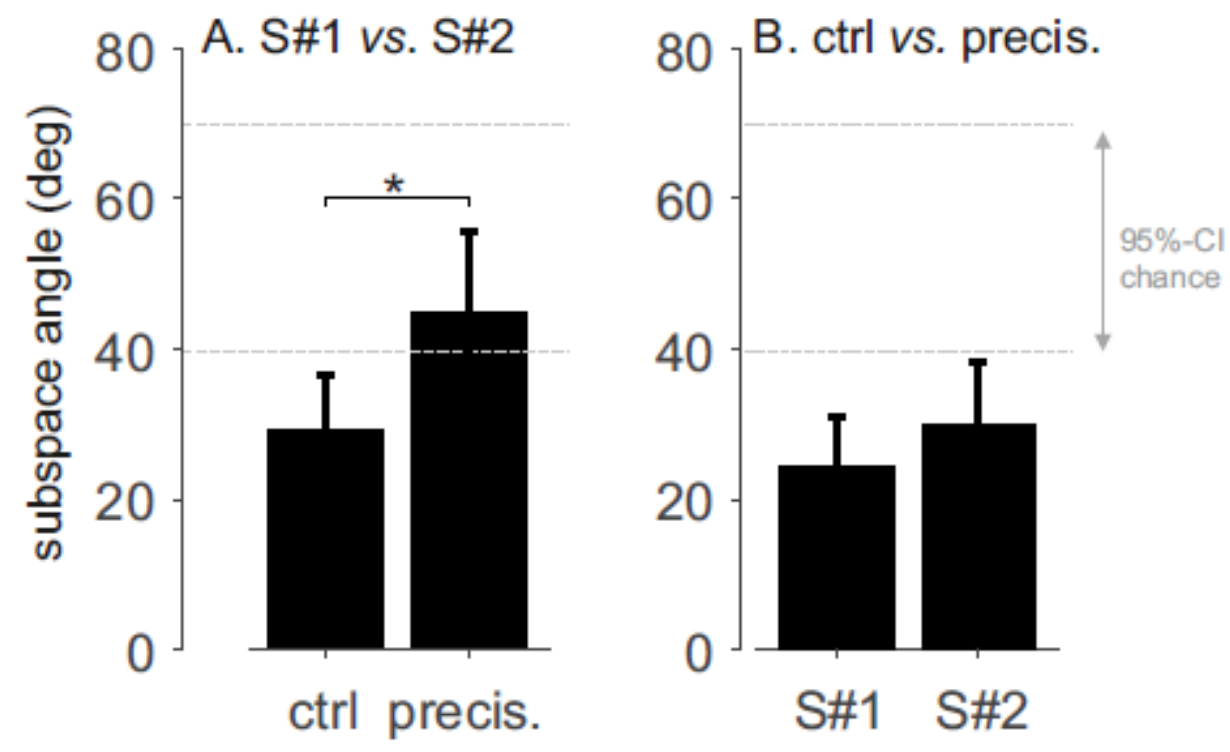

Figure 6. Subspaces comparison. The subspace angle measures the similarity between the subspaces
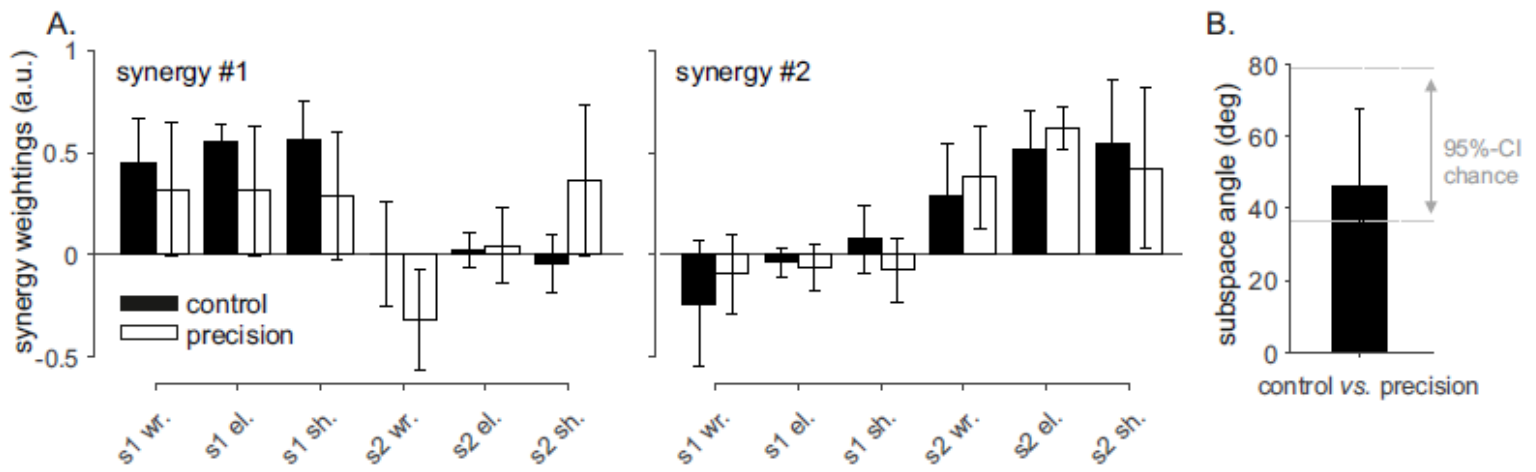

341 Figure 7. Conjoint synergies. S1 and S2 refer to participants \#1 (right side) and participant \#2 (left side), respectively. The 95\%-confidence interval of angles obtained with random synergies (95\%-CI 


\subsubsection{Moment Cost Function (MCF)}

346 In the CC the results obtained were divided into two different groups of dyads. Five dyads 347 showed higher values of both Total $M C F$ (Fig. 8A), $\left(339,35 \mathrm{~kg} \cdot \mathrm{m}^{2} \cdot \mathrm{s}^{-2} \pm 20,44\right)$ and $\triangle M C F$ (Fig. 348 8B) while five other dyads showed lower values, $\left(169,17 \mathrm{~kg} \cdot \mathrm{m}^{2} \cdot \mathrm{s}^{-2} \pm 17,06\right)$.

349 Regarding the PC, the results of Total and $\triangle \mathrm{MCF}$ (respectively $172,66 \mathrm{~kg} \cdot \mathrm{m}^{2} . \mathrm{s}^{-2} \pm 19,16 ; 5,78$ $\left.\mathrm{kg} \cdot \mathrm{m}^{2} \cdot \mathrm{s}^{-2} \pm 12,85\right)$ were less variable than in the CC.

\subsection{Added task}

\section{$352 \quad$ 4.2.1 Accuracy score based on the ball trajectory}

353 Regarding the ball trajectory, we found a correlation between the displacement of the handle and the ball on $\mathrm{X}$-axis (Medio-lateral axis). The same goes for the displacement on the $\mathrm{y}$-axis which represent the Postero-anterior axis (Fig. 4B). On the vertical Z-axis, the ball displacement and the handle are positively correlated.

357 Correlations were also computed in order to study the relationship between the ball trajectory and the sum of forces exerted by the subjects on the handles (Fig. 4C). Only two of ten dyads had a correlation on the X-axis; a positive correlation for the dyad 1 and a negative correlation

360 for the dyad 10. On the Y-axis five of ten dyads had a significant negative correlation compared

361 to the other three dyads who had a significant positive correlation. For the vertical Z-axis, nine of ten dyads had a positive significant correlation.

\subsubsection{Accuracy score}

364 The mean Scoreaccuracy was $80.45 \pm 23.66 \%$ during one gait cycle of the PACS in the PC condition.

\subsection{Head and Trunk}

367 Table 2 shows that when individuals had to keep the ball in the center of the target they turned 368 the upper part of their body towards the box. Indeed, across the CC and the PC condition the orientation towards the box increased by 57.42, 9.22 and 3.29 degrees for the head, shoulders and pelvis, respectively. Also, the distance FOR-STE decreased by $7.69 \mathrm{~cm}$ between the CC and PC conditions, showing that the subjects were gazing at the box (Table S5). 


\section{Discussion}

373 In this experiment, 10 dyads transported a load in two different conditions: a Control Condition

374 (CC), in which they walked together while transporting a load, and a Precision Condition (PC),

375 in which they walked together while transporting a load and maintaining at the same time a ball

376 on its top. The first objective of our study was to test the hypothesis that the gait performance

377 of two individuals walking together while transporting a load (CC) is disturbed when a precision

378 task is added (PC).

379 We studied the center of mass of the system formed by the paired subjects and the box they

380 carried. The result showed that the CoMPACS speed decreased when the precision constraint was

381 added. Besides, the second task induced a decrease in the pendular behavior and amplitude of

382 the system. However, the period of the CoMPACs displacement was not affected in PC. These

383 results could be expected as the added task can be considered as a fine motor skill (Exner,

384 2001). Indeed, and as observed for the gait of an adult performing a dual-task (Yogev-

385 Seligmann et al., 2010), the individuals needed to reduce their speed to perform both tasks.

386 Similar to the findings of Holt et al., (2003) in a single carrier, the decrease in speed was

387 accompanied by a decrease in the CoMpACs vertical amplitude. These adaptations are

388 reminiscent of the classic speed-accuracy tradeoff and the reduction in speed is very likely a

389 strategy to reduce motor noise and improve controllability.

390 To explore the task further, we also studied each subject as a distinct entity. The comparison of 391 the individual CoM trajectory of both subjects for CC and PC did not show any significant 392 difference. Indeed, when walking side-by-side with a sensory interaction subject tend to 393 synchronize their walking pace and kinematics (Schmidt and Turvey, 1995; Zivotofsky et al., 394 2012). The same goes for the CoM parameters (velocity, and CoM amplitude), i.e., no 395 significant differences were found between the subjects at the left and the one at the right. The 396 comparison of joint angles showed only a significant difference in hip angles between subjects.

397 No matter the condition they performed, the subjects tend to synchronize their speed and gesture 398 frequency. However, the precision task altered the CoMpacs kinematics leading to a less 399 efficient energy transfer. In fact, this spatio-temporal strategy induced a decrease of the 400 pendulum-like behavior at the CoMPACs.

401 Here, the energy recovered $(R R)$ values obtained for the PACS in the CC (mean $\pm \mathrm{CI}_{0.95}=60.25$ $402 \pm 8.57 \%$ ) were similar to those obtained in single carriers alone, as measured by Bastien et al. 
(2016) in Nepalese porters and in untrained individuals $(R R=61 \%)$, or by Tesio et al., (1998) in healthy individuals $(R R=60 \%)$. Our results showed a significant RR decrease in PC. This confirms the CoMpacs pendulum-like behavior alteration. The potential and kinetic energy being out of phase, RR decrease leads to a higher mechanical cost of the whole system due to the precision task. On the other hand, the global muscular efforts estimated thanks to the moment cost function (MCF) of the upper-limbs were much more balanced between the individuals of each dyad when they performed the dual-task than in the lifting condition (Fig $8)$.

Doi et al., (2011) demonstrated that increasing the difficulty of a task (e.g., dual-task) affects the cost of the movement in elderly adults. Similarly, we found a modification in the trunk posture in PC that might have resulted in a finer control of the task. During PC, subjects spontaneously oriented their head, shoulders, and pelvis towards the box, probably to gather more visual information. Modification on the orientation of the upper part of the body seems to allow the subjects to look at the ball on the top of the load but, at the same time, these body segments were locked in a position that likely disturbed the kinematic of the PACS and his ability to behave as a pendulum. This interpretation is in accordance with the findings of Winter (1995), who suggested that during a bipedal walking, the control of the trunk restrains vision and head control. Here, the swings and rotations of these segments (head, shoulders, and pelvis),

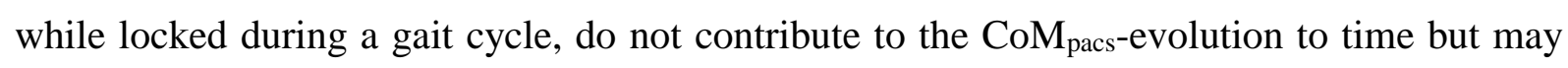
explain the lower-pendulum-like behavior in the PC. The decrease of the vertical amplitude of the CoMPACS with both trunk and head fixed to look at the ball reveals a lower limbs pattern altered in PC. Numerous research studied the impact of the trunk posture on gait pattern, whether for medical purposes (Moraud et al., 2018) or sport performance purposes (Teng and Powers, 2014; Huang et al., 2019). These studies showed that a modification of trunk posture on the frontal and sagittal plane influences the bilateral lower limb kinematics and muscle activity. Here, the control of the walking speed and of the ball may have been supported by the lower legs and induced the dissipation of the mechanical energy thanks to eccentric work of the muscles.

The impact of the added task on the physical action of the subjects during the load transport was investigated. We recorded the forces applied by the subjects on the two box handles (Fig 4) during CC and PC. The subjects' coordination was investigated through the correlation coefficient of applied forces. The results showed, in both conditions, a positive correlation for the forces on the antero-posterior and vertical axis. However, the correlation on the medio- 
lateral axis was weaker, with a large variability between the dyads of individuals. Thus, it seemed that the individuals coordinated their forces to move the load in the up-down and forward directions, without adopting a common strategy for the left-right direction. The results were similar between the two conditions, showing that the second task did not affect the collective strategies used during a simple load carriage task.

We considered that an action-reaction strategy was involved when the lag was higher than 150 $\mathrm{ms}$, which corresponds to the minimum latency observed to take a decision after the perception of a stimulus (VanRullen and Thorpe, 2001). All lags were lower than $150 \mathrm{~ms}$ in the mediolateral and antero-posterior axis. On the vertical axis however, lags higher than $150 \mathrm{~ms}$ were found in one dyad in the CC and in five dyads in the PC. Therefore, a modification of the behavior for half of the dyads was observed when the second task, requiring accuracy and precision, was added. These results might suggest a more conscious control of the box. It seems that the individuals moved the ball essentially by applying forces on the vertical axis at the handle inducing the rotation of the box around its anteroposterior axis and the displacement of the ball along the declination. When the box was thus moved by one individual, the second individual reacted (with a reaction time $>150 \mathrm{~ms}$ ) by moving it in the same direction in order to keep the ball in the center of the target.

Then, we used the moments applied on the box's' handles to compute through an inverse dynamic method the constraints at the joints of both upper-limbs of each subject. We observed a large variability of the joint moments among dyads in the CC. Half of the dyads produced much greater efforts than the other half and within this half, the joint moments produced by each individual were very unbalanced. In the precision condition, each individual within these five dyads produced similar efforts to keep the ball inside the circular target and to displace the load during a whole gait cycle. In both conditions, the participants were not allowed to communicate. However, in order to maintain the ball inside the target in the PC the dyad had to gaze at the box. Research on collective tasks showed that when sharing visual information's of their performance, group members tend to coordinate their forces and movements (Bosga and Meulenbroek, 2007; Schmidt et al., 1998; Schmidt and Turvey, 1995). Hence, the visual feedback could have an impact on the muscular effort variability between dyads, as well as between individuals within each dyad.

These results suggest that the load carriage was affected by the second task, independently of the individuals' performances in this task. Indeed, the accuracy score was not correlated 
( $\mathrm{r}=0.31, \mathrm{p}=0.386$ ) to the $R R$. The accuracy score was high, suggesting a good investment of the participants in the second task. According to Yogev-Seligmann et al., (2008) walking is a complex motor activity, which requires both the mobilization of executive functions, i.e. the cognitive capacities that allow an immediate adaptation of the motor behavior, and precision. On one hand, the decrease in locomotor performance in the precision condition could be explained by an increase in precision and a decrease in the mobilization of the executive functions used in locomotion. In the other hand, it also could be explained by a strategy of prioritization due to a structural interference between the precision needed to realize the first and the second task. Ebersbach et al., (1995), concluded that even when a task is highly practiced (e.g walking), adding concurrent tasks would lead to strategy changes depending on the attentional demand. Indeed, these control strategies are commonly used for humanoid robot when generating a movement prioritization. Sentis and Khatib, (2005), proposed a multi-level control hierarchy where the global task is decomposed into several subtasks. The hierarchy used ensured that constraints and critical task where accomplished first, while optimizing the execution of the global task. However, the absence of correlation between the accuracy score and the RR value reveals that the precision task may be too easy and may not discriminate different levels of precision.

Concerning the kinetic synergy analysis, a first observation is the non symmetry in terms of vector weightings for the loaded and unloaded arms i.e., the wrist, elbow and shoulder joint moments co-vary more with the neck and back joint moments for the loaded arm than for the unloaded arm, as demonstrated by high weightings coefficients for these joints (Fig 5). Subjects used more similar synergies during the $\mathrm{CC}$ than during the PC (Fig. 6A). The conjoint synergies were less similar than expected by chance when comparing the control and precision conditions (Fig. 7B). These two results show that a change in inter-joint moments coordination occurred due to the precision constraint. The synergies appeared more variable during the precision condition (Fig 5) and the weighting coefficients were "shared" between participants during the precision condition, e.g., the wrist joint moment for subject \#2 was loaded with the wrist, elbow, and shoulder joint moment of subject \#1 in the first conjoint synergy (Fig. 7A). These results suggest that although the coordination was more variable during the precision condition more co-variation occurred between the joint moments of the two participants. These results show that the collaboration during the precision task required disorganization of the spontaneous coordination adopted by the participants when no precision constraint was present. The change in posture between CC and PC might partly explain this observation. The VAF for the conjoint 
501 analysis tended to be lower during the precision condition (i.e., $\mathrm{p}=0.06$ ) also suggesting a more

502 variable coordination pattern between the joint moments of the two participants. These results

503 suggest that coordinated action between two subjects does not necessarily require a similar

504 coordination pattern for each of them, i.e., similar dynamic synergies, and that, in our

505 experiments, a new coordination pattern emerged between the two subjects (i.e., different joint

506 synergies), with more co-variation between their joint moments.

507 Our study highlights the fact that, when a dyad of individuals collectively transports a load and

508 performs a second task (requiring accuracy and precision), the displacement of the CoM of the

509 whole system (PACS) is affected, inducing a less efficient pendulum-like behavior. In both

510 conditions the individuals coordinated their forces to move in the vertical and forward direction

511 without adopting a common strategy in the left-right direction. We also observed that the

512 individuals changed their trunk orientation and their behavior to manage the displacement of

513 the ball inside the target. Furthermore, the visual feed-back permitted the dyad to coordinate

514 their forces and movements in order to better control the position of the ball. However, the

515 kinetic synergy analysis showed that subjects altered the structure of their own synergies in PC

516 to adopt a coordination that was dissimilar between subjects but in which their wrist joint

517 moments co-varied more. These results could be of interest for people working in ergonomics

518 and could find potential developments in robotics (e.g. human-robot interactions), and in the

519 rehabilitation domain, for example, when several caregivers in health care establishments have

520 to move a patient. 


\section{$521 \quad 6 \quad$ List of symbols and abbreviations}

522 CC: Control condition

523 CoM: Center of mass

524 CI: Confidence Interval

525 MCF: Moment Cost Function

526 PACS: Poly-Articulated Collective System

527 PC: Precision Condition

528 PCA: Pincipal Component Analysis

529 RR: Recovery Rate

530 VAF: Variance Accounted For

\section{$531 \quad 7 \quad$ Author contributions}

532 NS: responsible for the data analysis, interpretation and manuscript writing.GF: responsible for

533 th edata analysis and interpretation, and major revisions of the manuscript. VF: responsible for

534 the study design, supervision, data interpretation and major revisions of the manuscript. NAT:

535 responsible for the data analysis and interpretation and major revisions of the manuscript. PM:

536 Responsible for the study design, supervision, data interpretation and major revisions of the 537 manuscript.

\section{$538 \quad 8 \quad$ Competing interests}

539 The authors do not have to disclose any financial or personal relationships with other people or 540 organizations that could inappropriately influence (bias) their work.

\section{Funding}

542 This work was supported by the Financial support was provided by the Agence Nationale de la

543 Recherche [CoBot-Projet-ANR-18-CE10-0003], the Association Nationale Recherche 544 Technologie [CIFRE 2015/1321], and the MAS Marquiol for G.F. PhD grant. 
Ackerman, J., and Seipel, J. (2014). A model of human walking energetics with an elasticallysuspended load. Journal of Biomechanics, 47(8), 1922-1927.

Barnekow-Bergkvist, M., Aasa, U., Ängquist, K.-A., and Johansson, H. (2004). Prediction of development of fatigue during a simulated ambulance work task from physical performance tests. Ergonomics, 47(11), 1238-1250.

Bastien, G. J., Willems, P. A., Schepens, B., and Heglund, N. C. (2016). The mechanics of head-supported load carriage by Nepalese porters. The Journal of Experimental Biology, 219(Pt 22), 3626-3634.

Beach, T. A. C., Coke, S. K., and Callaghan, J. P. (2006). Upper body kinematic and low-back

Bosga, J., and Meulenbroek, R. G. J. (2007). Joint-Action Coordination of Redundant Force Contributions in a Virtual Lifting Task. Motor Control, 11(3), 235-258.

Castillo, E. R., Lieberman, G. M., McCarty, L. S., and Lieberman, D. E. (2014). Effects of pole compliance and step frequency on the biomechanics and economy of pole carrying during human walking. Journal of Applied Physiology, 117(5), 507-517.

Cavagna, G. A., Saibene, F. P., and Margaria, R. (1963). External work in walking. Journal of Applied Physiology, 18(1), 1-9.

Claudino, J. G., Gabbett, T. J., Bourgeois, F., Souza, H. de S., Miranda, R. C., Mezêncio, B., Soncin, R., Cardoso Filho, C. A., Bottaro, M., Hernandez, A. J., Amadio, A. C., and Serrão, J. C. (2018). CrossFit Overview: Systematic Review and Meta-analysis. Sports

Costes, A., Turpin, N. A., Villeger, D., Moretto, P., and Watier, B. (2018). Spontaneous change from seated to standing cycling position with increasing power is associated with a minimization of cost functions. Journal of Sports Sciences, 36(8), 907-913.

de Leva, P. (1996). Adjustments to Zatsiorsky-Seluyanov's segment inertia parameters. Journal of Biomechanics, 29(9), 1223-1230.

Doi, T., Asai, T., Hirata, S., and Ando, H. (2011). Dual-task costs for whole trunk movement during gait. Gait \& Posture, 33(4), 712-714. 
Ebersbach, G., Dimitrijevic, M. R., and Poewe, W. (1995). Influence of Concurrent Tasks on Gait: A Dual-Task Approach. Perceptual and Motor Skills, 81(1), 107-113.

Exner, C. E. (2001). Development of hand skills. Occupational Therapy for Children, 5, 304355.

Faber, G. S., Kingma, I., and Dieën, J. H. V. (2007). The effects of ergonomic interventions on low back moments are attenuated by changes in lifting behaviour. Ergonomics, 50(9), 1377-1391.

Faber, G., Visser, S., Van der Molen, H. F., Kuijer, P. P. F. M., Hoozemans, M. J. M., Van Dieën, J. H., and Frings-Dresen, M. H. W. (2012). Does team lifting increase the variability in peak lumbar compression in ironworkers? Work, 41, 4171-4173.

Fumery, G., Claverie, L., Fourcassié, V., and Moretto, P. (2018). Walking pattern efficiency during collective load transport. Gait \& Posture, 64, 244-247.

Gamble, R. P., Stevens, A. B., McBrien, H., Black, A., Cran, G. W., and Boreham, C. A. (1991). Physical fitness and occupational demands of the Belfast ambulance service. Occupational and Environmental Medicine, 48(9), 592-596.

Godwin, A. A., Stevenson, J. M., Agnew, M. J., Twiddy, A. L., Abdoli-Eramaki, M., and Lotz, C. A. (2009). Testing the efficacy of an ergonomic lifting aid at diminishing muscular fatigue in women over a prolonged period of lifting. International Journal of Industrial Ergonomics, 39(1), 121-126.

Heglund, N. C., Willems, P. A., Penta, M., and Cavagna, G. A. (1995). Energy-saving gait mechanics with head-supported loads. Nature, 375(6526), 52-54.

Holt, K. G., Wagenaar, R. C., LaFiandra, M. E., Kubo, M., and Obusek, J. P. (2003). Increased musculoskeletal stiffness during load carriage at increasing walking speeds maintains constant vertical excursion of the body center of mass. Journal of Biomechanics, 36(4), 465-471. 
Huang, Y., Xia, H., Chen, G., Cheng, S., Cheung, R. T. H., and Shull, P. B. (2019). Foot strike pattern, step rate, and trunk posture combined gait modifications to reduce impact loading during running. Journal of Biomechanics, 86, 102-109.

Knyazev, A. V., and Argentati, M. E. (2002). Principal Angles between Subspaces in an ABased Scalar Product: Algorithms and Perturbation Estimates. SIAM Journal on Scientific Computing, 23(6), 2008-2040.

Leyk, D., Rohde, U., Erley, O., Gorges, W., Essfeld, D., Erren, T. C., and Piekarski, C. (2007). Maximal manual stretcher carriage: performance and recovery of male and female ambulance workers. Ergonomics, 50(5), 752-762.

Moraud, E. M., von Zitzewitz, J., Miehlbradt, J., Wurth, S., Formento, E., DiGiovanna, J., Capogrosso, M., Courtine, G., and Micera, S. (2018). Closed-loop control of trunk posture improves locomotion through the regulation of leg proprioceptive feedback after spinal cord injury. Scientific Reports, 8(1), 76.

Moretto, P., Villeger, D., Costes, A., and Watier, B. (2016). Elastic energy in locomotion: Spring-mass vs. poly-articulated models. Gait \& Posture, 48, 183-188.

Osoba, M. Y., Rao, A. K., Agrawal, S. K., and Lalwani, A. K. (2019). Balance and gait in the elderly: A contemporary review. Laryngoscope Investigative Otolaryngology, 4(1), $143-153$.

Pashler, H. (1994). Dual-task interference in simple tasks: Data and theory. Psychological Bulletin, 116(2), 220-244.

Restorff, W. V. (2000). Physical fitness of young women: carrying simulated patients. Ergonomics, 43(6), 728-743.

Schmidt, R. C., Bienvenu, M., Fitzpatrick, P. A., and Amazeen, P. G. (1998). A comparison of intra- and interpersonal interlimb coordination: Coordination breakdowns and coupling strength. Journal of Experimental Psychology: Human Perception and Performance, 24(3), 884-900.

Schmidt, R. C., and Turvey, M. T. (1995). Models of interlimb coordination-Equilibria, local analyses, and spectral patterning: Comment on Fuchs and Kelso (1994). Journal of Experimental Psychology: Human Perception and Performance, 21(2), 432-443. 
Sentis, L., and Khatib, O. (2005). Synthesis of whole-body behaviors through hierarchical control of behavioral primitives. International Journal of Humanoid Robotics, 02(04), 505-518.

Sharp, M. A., Rice, V. J., Nindl, B. C., and Williamson, T. L. (1997). Effects of Team Size on the Maximum Weight Bar Lifting Strength of Military Personnel. Human Factors, $39(3), 481-488$.

Teng, H.-L., and Powers, C. M. (2014). Sagittal Plane Trunk Posture Influences Patellofemoral Joint Stress During Running. Journal of Orthopaedic \& Sports Physical Therapy, 44(10), 785-792.

Tesio, L., Lanzi, D., and Detrembleur, C. (1998). The 3-D motion of the centre of gravity of the human body during level walking. I. Normal subjects at low and intermediate walking speeds. Clinical Biomechanics, 13(2), 77-82.

VanRullen, R., and Thorpe, S. J. (2001). The Time Course of Visual Processing: From Early Perception to Decision-Making. Journal of Cognitive Neuroscience, 13(4), 454-461.

Willems, P. A., Cavagna, G. A., and Heglund, N. C. (1995). External, internal and total work in human locomotion. Journal of Experimental Biology, 198(2), 379-393.

Winter, D. (1995). Human balance and posture control during standing and walking. Gait \& Posture, 3(4), 193-214.

Wu, G., Siegler, S., Allard, P., Kirtley, C., Leardini, A., Rosenbaum, D., Whittle, M., D’Lima, D. D., Cristofolini, L., Witte, H., Schmid, O., and Stokes, I. (2002). ISB recommendation on definitions of joint coordinate system of various joints for the reporting of human joint motion — part I: ankle, hip, and spine. Journal of Biomechanics, 35(4), 543-548.

Wu, G., van der Helm, F. C. T., (DirkJan) Veeger, H. E. J., Makhsous, M., Van Roy, P., Anglin, C., Nagels, J., Karduna, A. R., McQuade, K., Wang, X., Werner, F. W., and Buchholz, B. (2005). ISB recommendation on definitions of joint coordinate systems of various joints for the reporting of human joint motion-Part II: shoulder, elbow, wrist and hand. Journal of Biomechanics, 38(5), 981-992.

Yogev-Seligmann, G., Hausdorff, J. M., and Giladi, N. (2008). The role of executive function and attention in gait. Movement Disorders, 23(3), 329-342. 
664 Yogev-Seligmann, G., Rotem-Galili, Y., Mirelman, A., Dickstein, R., Giladi, N., and Hausdorff, J. M. (2010). How Does Explicit Prioritization Alter Walking During DualTask Performance? Effects of Age and Sex on Gait Speed and Variability. Physical Therapy, 90(2), 177-186.

668 Zatsiorsky, V. (1983). The mass and inertia characteristics of the main segments of the human 669 body. Biomechanics, 1152-1159.

670 Zivotofsky, A. Z., Gruendlinger, L., and Hausdorff, J. M. (2012). Modality-specific 671 communication enabling gait synchronization during over-ground side-by-side walking. Human Movement Science, 31(5), 1268-1285. 


\section{Figure legends}

674 Figure 1. Experimental setup: Collective load carriage performed with a precision task 675 (PC). The dyad carried a box (mass $=13.41 \mathrm{~kg}$, size: $0.40 \mathrm{x} 0.40 \times 0.28$ ) while maintaining a 676 ball (diameter $=19 \mathrm{~mm}$, mass $=2 \mathrm{~g}$ ) in the center of a targeted position (diameter of the small and 677 large circle: $120 \mathrm{~mm}$ and $240 \mathrm{~mm}$ respectively) on its top. If the ball was maintained in the 678 small circle, the dyad obtained a 1-point Score . If it was maintained between the small and 679 large circle, then they obtained 0,5-point Score p $^{\text {Else, they obtained 0-point Score }}$.

Figure 2. Amplitude (A) and period (B) of the vertical displacement of the CoMpacs in the 682 Control Condition (CC) and the Precision Condition (PC). The mean value of each dyad 683 ( $\mathrm{N}=10)$ was computed for the $\mathrm{CC}$ and PC and linked. The red points represent the mean for 684 each condition. The same color is assigned to each dyad in all figures. $*=$ significant difference 685 $(\mathrm{p}<0.05$ paired t-test).

Figure 3. Recovery Rate (\%) of each dyad (N=10) during Control Condition (CC) and Precision Condition (PC). The red points represent the mean for each condition. The same color is assigned to each dyad in all figures. $*=$ significant difference $(\mathrm{p}<0.05$ paired $\mathrm{t}$-test $)$.

690

692 of the forces produced by the individuals in each dyad on the box handles, on the medio-lateral 693 (Fx), antero-posterior (Fy) and vertical axis (Fz) in the CC and PC conditions. B : Coef of the 694 ball displacement and the handles displacement, on Fx, Fy, and Fz, in the CC and PC conditions. $695 \mathrm{C}$ : Coef of the ball trajectory and the sum of forces exerted by the subjects on the handles, on 696 Fx, Fy, and Fz, in PC. $\mathrm{N}=10$ for each condition. * 0.05> p > 0.01; ** p <0.01 (paired Student $697 \mathrm{t}$ test). The upper horizontal line of the box represents the third quartile (75th percentile), the 698 lower line of the box represent the first quartile (25th percentile), the middle value of the dataset 699 is the median value (50th percentile) and the upper and lower horizontal lines outside the box 700 represent respectively 90th percentile and 10th percentile. Cross-and circle represent 701 respectively outlier and mean. 
703 Figure 5. Dynamic synergy vectors. Three synergies accounted for more than $90.0 \%$ of total 704 variance in all subjects and conditions. Participants labeled \#1 were on the right side of the load 705 and participants \#2 on the left side. Wrist, elbow and shoulder were abbreviated to wr. el. and 706 sh. respectively. $\mathrm{R}$ and $\mathrm{L}$ refer to right and left side, respectively.

707

708 Figure 6. Subspaces comparison. The subspace angle measures the similarity between the 709 subspaces spanned by the dynamic synergies. The 95\%-confidence interval of angles obtained 710 with random synergies $\left(95 \%-\mathrm{CI}\right.$ chance) is indicated, i.e., $\mathrm{CI}=\left[49.5^{\circ}, 70.0^{\circ}\right]$. the $\operatorname{star}(*)$ 711 indicates a significant difference (i.e., $\mathrm{p}<0.001$ ).

713 Figure 7. Conjoint synergies. S1 and S2 refer to participants \#1 (right side) and participant \#2 714 (left side), respectively. The 95\%-confidence interval of angles obtained with random synergies $715\left(95 \%\right.$-CI chance) is indicated in panel B, i.e., $\mathrm{CI}=\left[36.3^{\circ}, 79.1^{\circ}\right]$. 


\begin{tabular}{ccccccc}
\hline \multirow{2}{*}{ Group } & \multicolumn{3}{c}{ Control Condition } & \multicolumn{3}{c}{ Precision Condition } \\
\cline { 2 - 7 } $\mathbf{1}$ & LagX & LagY & LagZ & LagX & LagY & LagZ \\
$\mathbf{2}$ & 0 & 0 & 0 & 0 & 0 & $0,155^{*}$ \\
$\mathbf{3}$ & 0 & 0 & 0,04 & 0 & 0 & $0,56 *$ \\
$\mathbf{4}$ & 0 & 0 & 0 & 0 & 0 & 0 \\
$\mathbf{5}$ & 0 & 0 & 0 & 0 & 0 & 0 \\
$\mathbf{6}$ & 0 & 0 & 0 & 0 & 0 & 0 \\
$\mathbf{7}$ & 0 & 0 & 0 & 0 & 0 & $0,15 *$ \\
$\mathbf{8}$ & 0 & 0 & $0,15 *$ & 0 & 0 & 0 \\
$\mathbf{9}$ & 0 & 0 & 0 & 0 & 0 & $0,355^{*}$ \\
$\mathbf{1 0}$ & 0 & 0 & 0 & 0 & 0 & 0 \\
\hline
\end{tabular}

717 Table 1. The action-reaction strategy, the time lag (s) required for the position of the left side 718 and right side of the box to be the same on the medio-lateral, antero-posterior and vertical axis 719 in CC and PC. * 150 ms $<$ p (VanRullen and Thorpe,2001)

\begin{tabular}{|ccccc}
\hline & CC & S.D & PC & S.D \\
\hline Head orientation & 2.58 & \pm 4.61 & $60.00 * *$ & \pm 11.81 \\
\hline Shoulders orientation & 1.99 & \pm 3.13 & $11.21 * *$ & \pm 6.61 \\
\hline Pelvis orientation & 0.16 & \pm 5.14 & $3.45 *$ & \pm 4.05 \\
\hline Distance FOR-STE & 21.67 & \pm 4.86 & $13.98 * *$ & \pm 3.25 \\
\hline
\end{tabular}

Table 2. Head, shoulders and pelvis orientation (angles in degrees) and distance between the forehead and the sternum (FOR-STE, in centimeters) in the CC and PC conditions; mean ( \pm s.d.). $\mathrm{N}=20$ for each condition. $* 0.05>\mathrm{p}>0.01 ; * * \mathrm{p}<0.01$ (paired Student $\mathrm{t}$ test). 